\title{
Cislunar Electron Content as Determined by Radar Group Delay Measurements
}

by

H. T. Howard
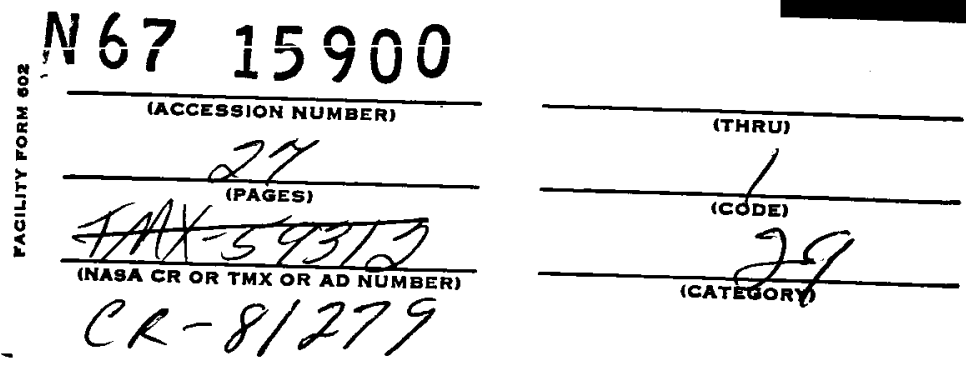

November 1966

$$
\begin{aligned}
& \text { GPO PRICE \$ } \\
& \text { CFSTI PRICE(S) \$ }
\end{aligned}
$$

Hard copy $(\mathrm{HC})$

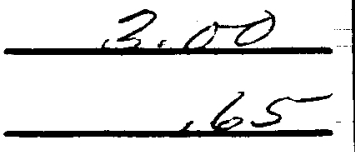

Scientific Report No. 17

Microfiche (MF)

Prepared under

National Aeronautics and Space Administration Grant NsG-377

\section{STAMFORD ELECTRONICS LABORATORIES}




\section{CISLUNAR ELECTRON CONTENT AS DETERMINED BY RADAR GROUP DELAY MEASUREMENTS}

by

H. T. Howard

November, 1966

Scientific Report No. 17

Prepared under

National Aeronautics and Space Administration

Grant NsG-377

Radioscience Laboratory

Stanford Electronics Laboratory

Stanford, California 
CISLUNAR ELECTRON CONTENT AS DETERMINED

BY RADAR GROUP DELAY MEASUREMENTS

\begin{abstract}
An earlier, preliminary report [Howard et al, 1965] described an experimental radar technique for measuring differential group delay to the moon with an accuracy of $10 \mu \mathrm{sec}$ and presented results of 5 measurements. Since that time, the 25 and $50 \mathrm{MHz}$ radars at stanford have been modified to improve power output and time resolution and, through the months of July and August, 1965, were used to determine cislunar electron content during 55 lunar meridian transits. Ionospheric electron contents, determined by the Faraday rotation on $136 \mathrm{mHz}$ signals from Syncom III were combined with calculated protonospheric values and subtracted from each radar determination of total content. The results of this experiment reinforce the earlier conclusion that there are two distinct regions beyond the plasmasphere. In the general direction of the sun where most of the radar path is beyond the shock wave boundary, the average electron number density is very low:
\end{abstract}


$11 \pm 30 \mathrm{~cm}^{-3}$. Within the solar wind wake of the earth, in the quadrant opposite the sun, the columnar content from approximately $4 R_{E}$ to the moon is $4.9 \pm 1.2 \times 10^{16}$ electrons $\mathrm{m}^{-2}$. If this content were spread evenly along the path, it would imply a number density of $120 \pm 30 \mathrm{~cm}^{-3}$. The wake density and possibly its shape appear to change on a day to day basis.

\section{Introduction}

Lunar radar measurements of group delay on two frequencies were used in December 1964 to deduce cislunar electron content in several directions relative to the sun. The conclusions drawn from the work [Howard et al, 1965] were that in the quadrant opposite the sun, or the region known as the magnetospheric tail, the average cislunar electron density above the ionosphere is as high as several hundred per cubic centimeter and that toward the sun the density is much lower.

The experimental technique is based on the fact that radar-signal round-trip time is related to the distance from the radar to the moon and the mean square plasma frequency over the radar path [Eshleman et al, 1960] by

$$
T=T_{0}\left(1+\frac{\overline{f_{o}^{2}}}{2 f^{2}}\right), f \gg f_{0}
$$

where $T$ is the propagation time, $T_{0}$ the free space delay time, $f$ the radar frequency, and $\overline{f_{0}^{2}}$ the mean square plasma frequency. The plasma frequency is related to the electron density through

$$
\mathbf{f}_{\mathrm{o}}^{2}=\mathrm{c}^{2} \mathbf{r}_{\mathrm{e}} \mathrm{N} / \pi=80.6 \mathrm{~N}
$$

where $r_{e}$ is the classical electron radius $\left(2.8178 \times 10^{-15}\right.$ meters), 
and $\mathrm{N}$ is the number of electrons per cubic meter.

It follows that for a round-trip time to the moon of $2.6 \mathrm{sec}$ and a radar frequency of $25 \mathrm{MHz}$, the extra delay caused by an average of 100 electrons $\mathrm{cm}^{-3}$ along the entire path is $16.8 \mu \mathrm{sec}$. Thus $\mathrm{T}_{\mathrm{o}}$ must be known to a precision of one part in $10^{6}$ if a meaningful comparison is to be made. This need for absolute precision can be circumvented if the measurement is simultaneously performed on two frequencies $f_{1}$ and $f_{2}$, so that a relative delay can be measured. The delay difference will then be

$$
T_{1}-T_{2}=T_{0} \frac{\overline{f_{0}^{2}}}{2}\left(\frac{1}{f_{1}^{2}}-\frac{1}{f_{2}^{2}}\right)
$$

For the stanford radars with $f_{1}=25 \mathrm{MHz}$ and $f_{2}=50 \mathrm{MHz}$, the differential delay $T_{1}-T_{2}$ caused by an average electron number density of $100 \mathrm{~cm}^{-3}$ along the cislunar path would be $12.6 \mu \mathrm{sec}$.

\section{$\underline{\text { Experiment Description }}$}

Both the December 1964 measurements and other radar work [Yoh et al, 1966] demonstrated that the solar and anti-solar cislunar regions are distinctly different; however, the accuracy and quantity of data available were insufficient to draw a detailed picture. For this reason, special exciter and receiver equipment was constructed based on frequency synthesis techniques developed by another group in the stanford Radioscience Laboratory [Barry and Fenwick, 1965].

Briefly, the two radars are simultaneously swept in frequency at the rate of $1 \mathrm{~Hz}$ every $10 \mu \mathrm{sec}$ over a $100 \mathrm{kHz}$ band. After transmitting in this mode for the approximate round-trip time of $2.6 \mathrm{sec}$, receivers 
are connected to the antenna for the same length of time. During this latter time the receiver local oscillators are swept in frequency in an identical manner to the transmit sweep. The resulting beat note between the lunar echo and the swept oscillator is recorded on magnetic tape. The tape is then re-recorded to compress the data by a factor of 32 before processing on a spectran spectrum analyzer.

A sample spectrum analyzer output for a 2 -hour run is shown in Fig. 1. The axis labeled "Relative Time Delay" is actually a combination of range, $R$, and range rate, $\dot{R}$. The reason for the skewed appearance is that the $24.9 \mathrm{MHz}$ curve in $R+\dot{R}$ while the $49.8 \mathrm{MHz}$ curve is $R-\dot{R}$. In reducing the data, $\dot{R}$ is computed to find $\Delta R$ or, when converted to time, $T_{1}-T_{2}$

With the signal strengths received and the resolution available in the spectrum analyzer, it is possible to scale differences with an accuracy of $\pm 2.5 \mu \mathrm{sec}$ for each point. In terms of columnar electron content, this is $\pm 0.8 \times 10^{16}$ electrons $\mathrm{m}^{-2}$, or when spread over the entire earth-moon path, a number density of $\pm 20 \mathrm{~cm}^{-3}$. Daily measurements can be made for about $1-1 / 2$ hours with approximately 15 scalings accomplished at 5-minute intervals.

The uncertainty caused by roughness of the lunar surface is considerably larger as can be seen from the second-to-second jaggedness of the individual echoes in Fig. 1. It is also felt that ionospheric roughness or blob structure accounts for longer term (minutes) variation in echo range. Using 15 measurements as an average number, the error contribution of the moon-medium-equipment combination is approximately $\pm 12 \mu \mathrm{sec}$ per measurement or $\pm 3.1 \mu \mathrm{sec}$. In terms of electron content 


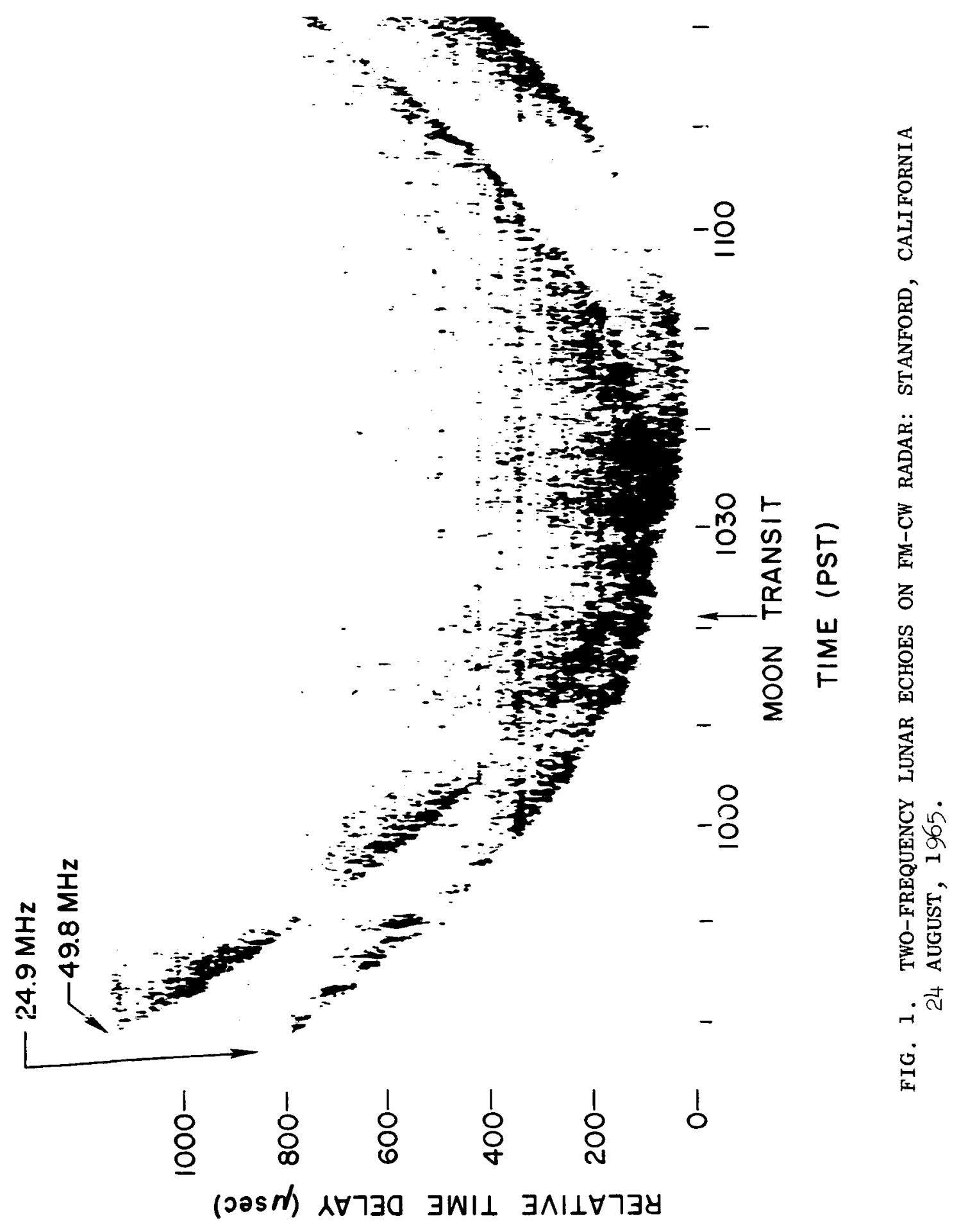


this is $\pm 25 \mathrm{~cm}^{-3}$ or $\pm 1 \times 10^{16}$ electrons $\mathrm{m}^{-2}$.

Recent criticism of this and other radar methods of measuring electron densities [Scarf, 1966] also needs to be considered. The argument has been advanced that coherent electron density fluctuations could severely modify propagation constants. On the other hand, Hagfors [1966] has demonstrated that, even in the unlikely circumstance that operating wavelength and density fluctuation scale size were to coincide, the total effect would be less than $1 \%$ and would be in the direction of decreasing radar measured content rather than in the increase originally postulated. Thus, it would appear that any such effect would be well masked by other measurement noise.

\section{Path Definition and Description}

For purposes of this paper the term ionosphere will be used to describe that region up to the transition between oxygen and lighter elements at roughly, $1000 \mathrm{~km}$. Above the transition region and to the plasmapause or "knee" will be called the protonosphere with due regard to the dominant role of ionized hydrogen. The entire region, combining ionosphere and protonosphere, is then called the "plasmasphere".

All of the discussion of densities to follow is based upon the radar path to the moon through the medium. This presentation distorts the usual vertical-content diurnal curve for the ionosphere but gives a much clearer picture of the remaining cislunar medium, as will be seen in the following sections. Since the plane of the lunar orbit is inclined to the ecliptic by approximately 5 degrees, the moon can be located above or below the ecliptic by about $5 R_{E}$ at its range of $60 R_{E}$. 
The intersection of the two planes is known as the line of the nodes and the position of the ascending node for the data period precessed from 0600 to 1000 local time. Thus, during the majority of this time, the moon was below the ecliptic when in the magnetospheric tail, with the maximum downward displacement located between the 0100 and 0300 directions. This means that the nighttime radar path penetrates the ecliptic plane at about $10 \mathrm{R}_{\mathrm{E}}$. For the period under discussion, the moon's elevation was a maximum $\left(77.6^{\circ}\right)$ between 1000 and 1200 local time and a minimum $\left(26.2^{\circ}\right)$ between 2200 and 2400 hours. Therefore, the path length through the earth's ionosphere was twice as long ( $\sec x=2$ for a 400-km 1ayer height) near midnight as $1 \mathrm{t}$ was near noon. The calculation of protonospheric content also takes this factor into account by stopping integration at the L-shell value equivalent to an equatorial knee position of $4 R_{E}$.

A diagram of the path geometry for both the day and night cases is presented in Fig. 2 as an aid to visualizing the relation between the radar path, the equatorial and ecliptic planes, and the plasmasphere.

\section{Ionospheric Measurement}

Since the radars measure content over the entire cislunar path, the plasmasphere's contribution must be subtracted if conclusions are to be made about the interplanetary and wake density. The ionospheric part of the path can be obtained in several ways, such as bottomside and topside sounding, radar Faraday, electron backscatter and various satellite Doppler and Faraday measurements. For this experiment, Faraday rotation on the $136 \mathrm{MHz}$ signals from Syncom III was used to deduce 


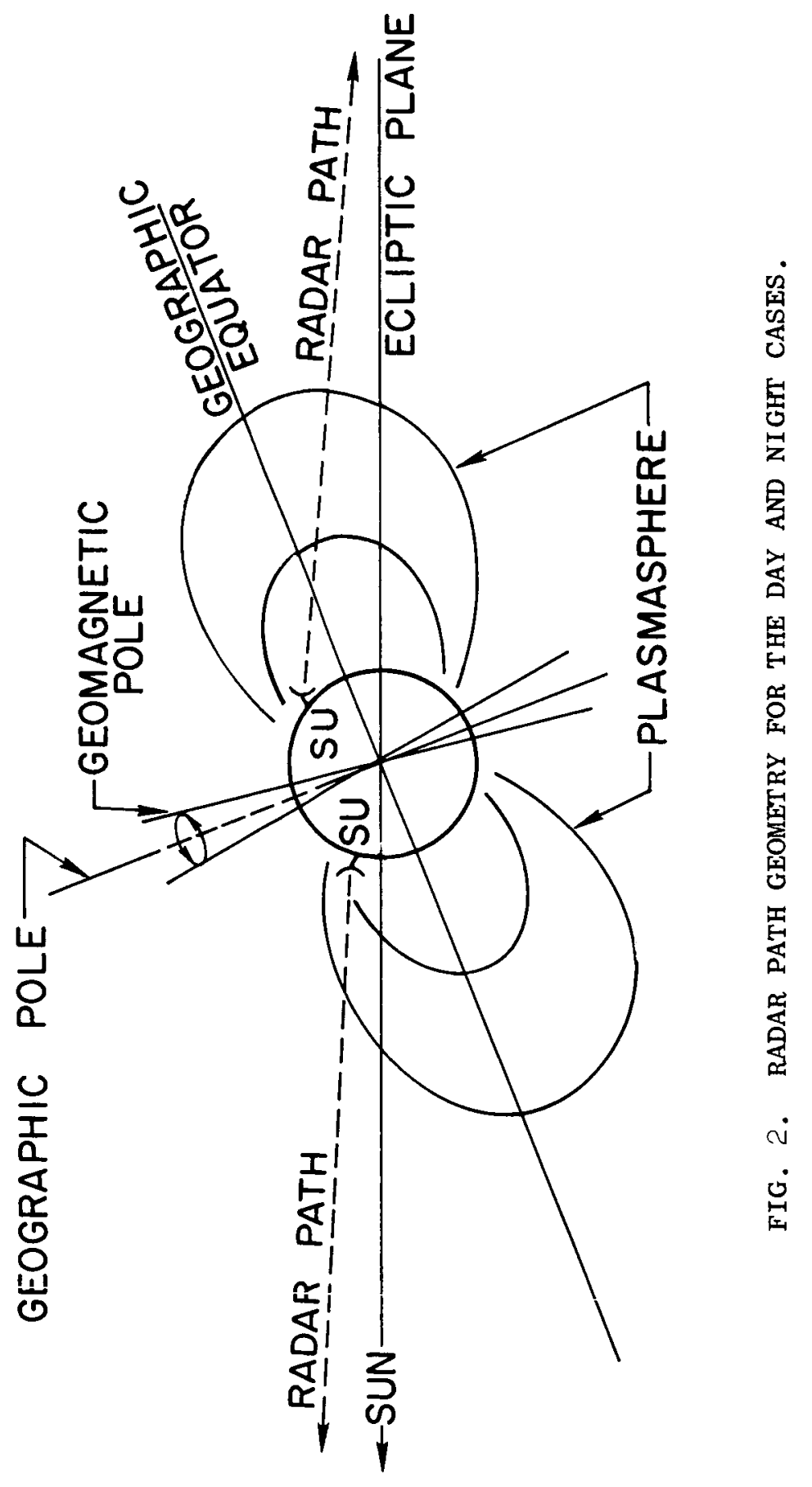


ionospheric content [Garriott et al, 1965]. The $n_{\pi}$ ambiguity in measuring polarization, equivalent to $3.96 \times 10^{16}$ electrons $\mathrm{m}^{-2}$, was removed by using the $\mathrm{C}-2$ vertical incidence ionosonde located on the Stanford campus.

Syncom was recorded continuously and an average content calculated for each period corresponding to the radar measurement time. These data were supplied [Smith, 1966] in the form of vertical columnar content at the subionospheric point which, during this time, was located at $33.6^{\circ} \mathrm{N}$ and $134.9^{\circ} \mathrm{W}$. Since the radar observations occurred around local moon transit time, the radar path subionospheric point varied from approximately $30^{\circ} \mathrm{N}$ to $36.5^{\circ} \mathrm{N}$ along the $121.5^{\circ} \mathrm{W}$ meridian. Longitudinal differences were minimized by comparing radar and syncom data at the subionospheric point local time. Detalls of the Syncom analysis are contained in the literature [Garriott et al, 1965]. It is important to note that though the integral extends along the entire path, it is heavily weighted at the lowest altitudes due to the $r^{-3}$ behavior of the earth's magnetic field and the rapid decrease in electron number density with height.

Comparison of these results with those obtained from Faraday measurements on S-66 at $1000 \mathrm{~km}$ shows that the Syncom Faraday method produces somewhat higher numbers, During the period under discussion 17 , s-66 passes analyzed averaged $10.2 \%$ less than the syncom contents. Nighttime passes are generally about $15 \%$ low while those taken during the day are within $5 \%$. This agrees well with the amount of rotation expected above $1000 \mathrm{~km}$ based on the protonospheric models discussed in the next section. Since the content above $1000 \mathrm{~km}$ is treated separ- 
ately, the Syncom Faraday contents have been adjusted downward by $1.2 \times 10^{16}$ electrons $\mathrm{m}^{-2}$ to agree better with ionosonde and Beacon satellite data. Analysis of the errors involved shows that the overall measurement accuracy expected is approximately 10 degrees in polarization angle or $\pm 0.4 \times 10^{16}$ electrons $\mathrm{m}^{-2}$.

\section{Protonospheric Measurement and Calculation}

In the previous work, no attempt was made to remove electron content between $1000 \mathrm{~km}$ and the plasmapause from the results. Since it is clear that this region can account for a significant fraction of the integrated content, two different techniques have been used to compute its contribution.

First, the columnar content has been calculated based on a diffusive equilibrium, hydrogen-only equatorial model [daRosa, 1966]. This model assumes an electron number density of $6 \times 10^{3} \mathrm{~cm}^{-3}$ at $1000 \mathrm{~km}$ and the total content is calculated along the radar path to the L-shell value for a $4 R_{F}$ knee position. It has been found that this content is relatively insensitive to knee position and is much more dependent upon temperature. Since not enough is presently known about the diurnal variation of $\mathrm{T}_{e}$ to choose a correct temperature for each time of day, a single value of $1500^{\circ} \mathrm{K}$ was selected as being reasonable in the antisolar direction. Total contents derived from this calculation are 2 and $3 \times 10^{16}$ electrons $\mathrm{m}^{-2}$ in the solar and ant1-solar directions, respectively.

A careful investigation of the effect of a fixed temperature and the hydrogen-only model has shown that the nighttime values are reasonable, and, if anything, high by $10 \%$. Conversely, the dayside values are 
not realistic for both the temperature and density assumed could be incorrect by a factor of two.

For this reason, a second approach was tried based on Alouette I data [Chan et al, 1966; Thomas et al, 1966] and excellent whistler data available for several days during the period [Angerami, 1966]. The night-side computation is again straightforward, for a similar model is assumed and the Alouette data yield an identical 1000-km content to that used previously. On the day side, however, a 1000-km electron number density of $25 \times 10^{3} \mathrm{~cm}^{-3}$ has been selected from the Alouette data as being representative. It should be noted that the most extensive data available are for Alouette passes taken at Stanford in the summer of 1963. These data have been compared with Ottawa data for the summers of 1963 and 1965 [Colin, 1966] and no consistent differences were found. In general, the densities appear relatively unchanged over the period of investigation and the total effect possible on the protonosphere calculation was less than $\pm 15 \%$. A further comparison with Explorer XXII Langmuir probe results [Brace, 1966] shows that the assumption of no significant change between 1963 and 1965 1000-km densities is well justified. Using the Alouette density and a somewhat more complete model, a computation of dayside content yields $3.05 \times 10^{16}$ electrons $\mathrm{m}^{-2}$. Thus, it has been concluded that, for the perlod in question, the protonospheric content was very close to $3 \times 10^{16}$ electrons $\mathrm{m}^{-2}$ along the radar path on both the night and day sides. This apparent independence of solar direction is caused by a large difference in radar path zenith angles from night to day. 


\section{Experimental Results}

In the following figures, a local time scale is used. This time can be interpreted as longitude in the geocentric solar ecliptic system as viewed from the north ecliptic pole since the data were taken a lunar meridian transit. Thus, noon is toward the sun and midnight is in the anti-solar direction. Error bars on individual plots reflect the preceding discussions of measurement and computation accuracy.

Figure 3 is a plot of the radar minus Syncom Faraday results for each period of operation. Starting with the first run on June 25, the content can be traced through two lunations by following first the solid line and then the dotted line ending on August 26 . From this figure it is very tempting to draw an analogy with the tail of a comet in the manner suggested by the IMP magnetometer [Ness, 1966]. The day-today and month variability are dominating features of this figure, while the difference in behavior between the solar and anti-solar directions is evident.

Figure 4 is the same daily data with the two months interlaced. The total spread is unchanged but the directional behavior is even more evident. Each daily point represents approximately $1-1 / 2$ hours of radar and Syncom data. The data points in this figure have been used as the basis for the remaining figures where a 5-point running mean is plotted for individual radar and ionospheric measurements.

Figure 5 presents the radar measured group delay expressed as earth-to-moon columnar content. For comparison purposes, the Syncom Ionospheric content and that calculated for the protonosphere are also shown. Comparison of the radar and syncom curves shows that much of the 


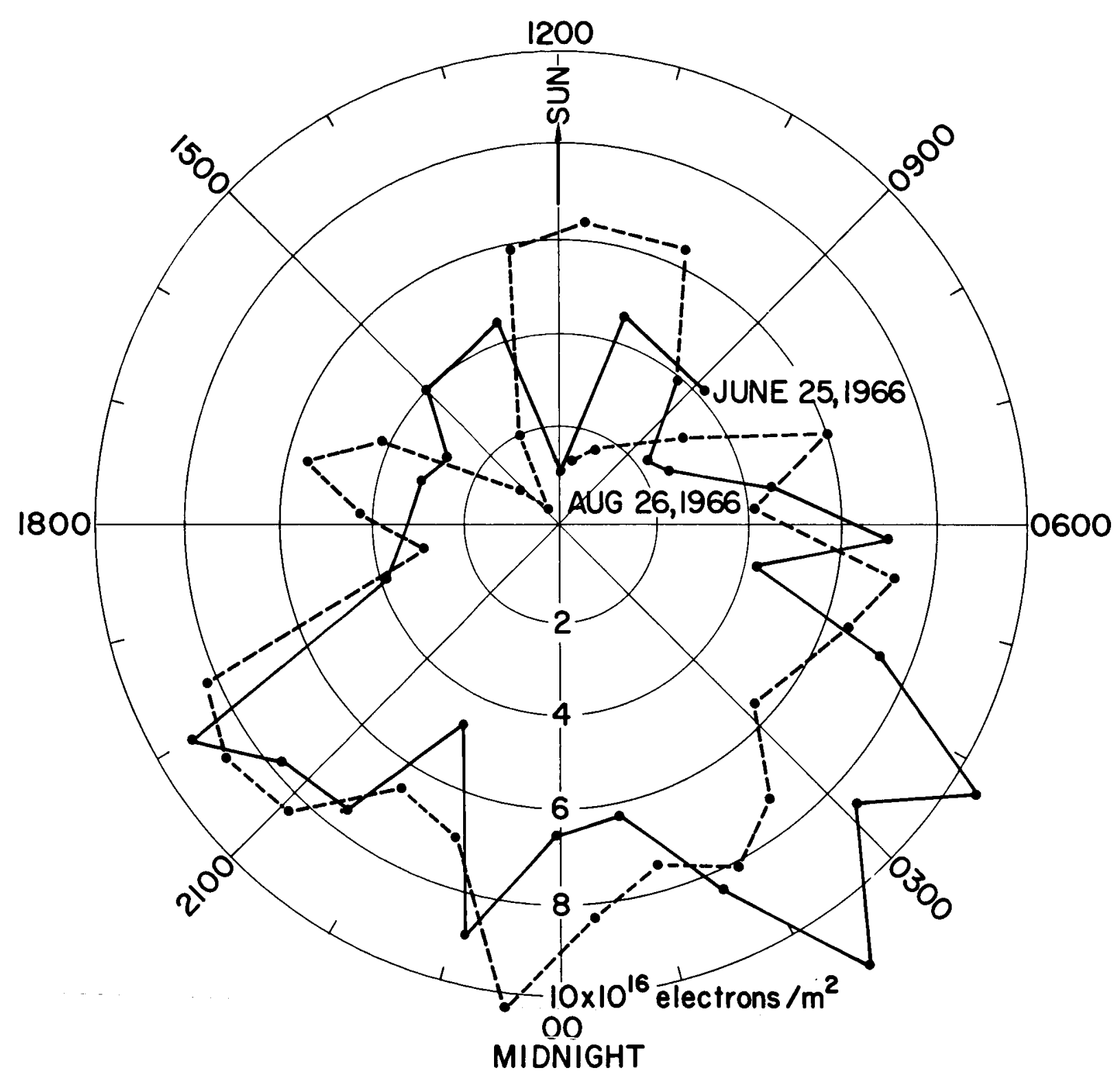

FIG. 3. RADAR GROUP DELAY ELECTRON CONTENT MINUS SYNCOM FARADAY ELECTRON CONTENT. Each dot represents approximately 1-1/2 hours of data taken around lunar meridian transit. 


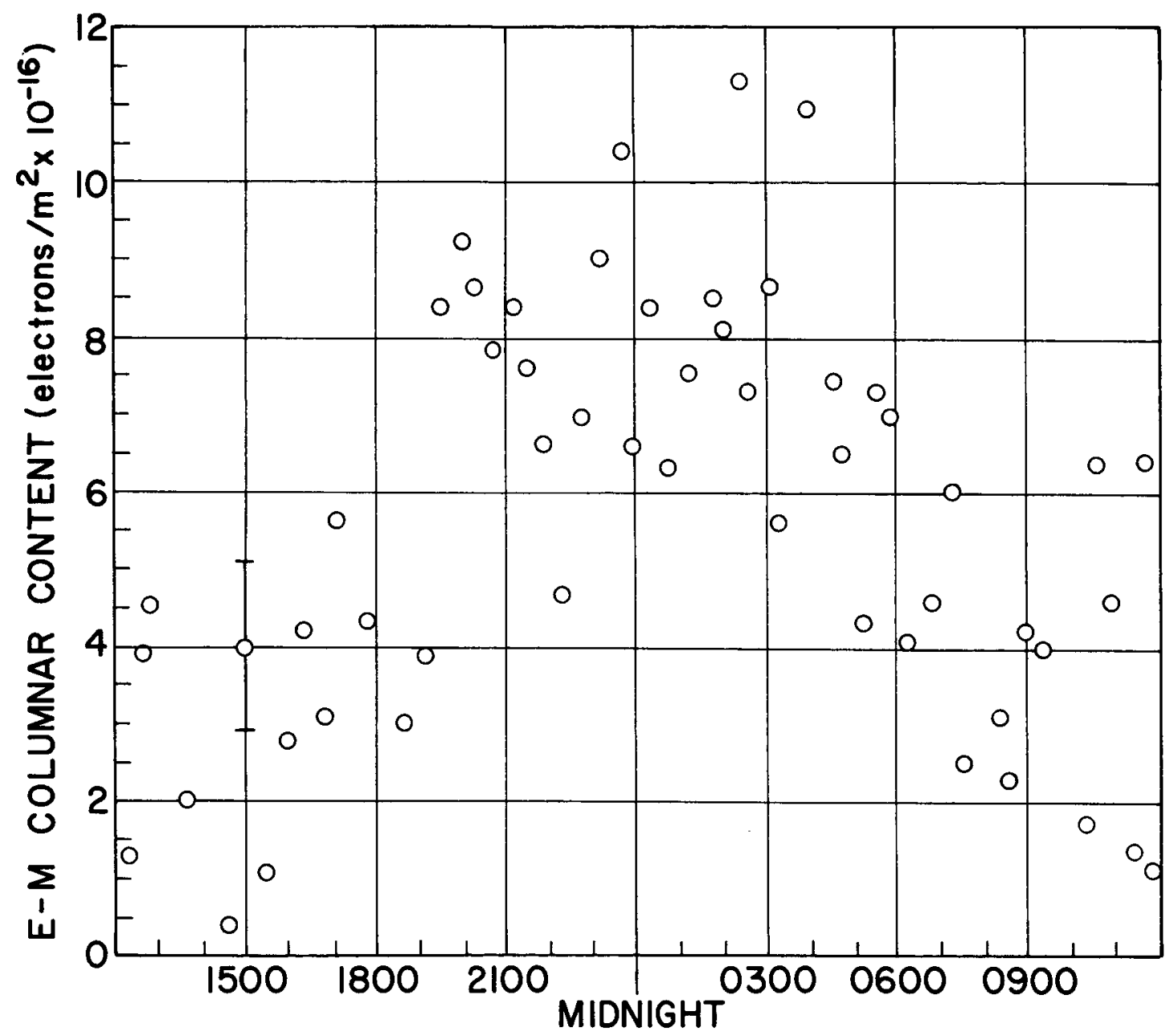

FIG. 4. RADAR GROUP DELAY ELECTRON CONTENT MINUS SYNCOM FARADAY ELECTRON CONTENT WITH ONE DATA DOINT FOR EACH DAY AND TWO MONTHS OF DATA INTERLACED. 


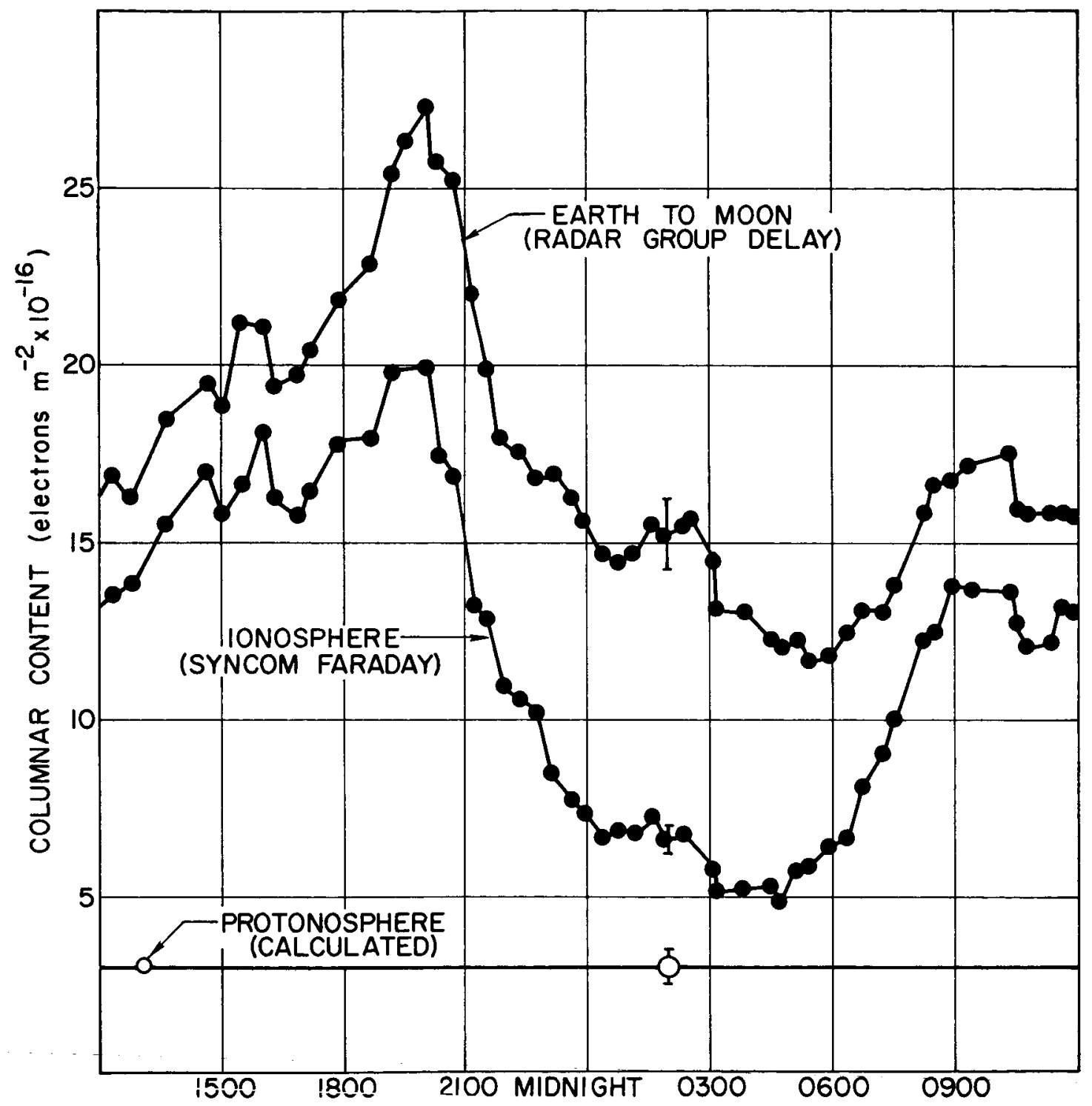

FIG. 5. FIVE DAY RUNNING MEAN OF COLUMNAR ELECTRON CONTENTS AS MEASURED BY RADAR GROUP DELAY AND FARADAY ROTATION OF SYNCOM SATELLITE SIGNALS. Calculated protonosphere content is plotted at $3.05 \times 10^{16}$ electrons $\mathrm{m}^{-2}$. 
detailed behavior is due to the ionosphere while there is a gradual increase in spread between them, beginning at 1500 , reaching a maximum just after midnight and decreasing until 0900. It should again be emphasized that the values plotted are those along the path, not vertical contents.

Figure 6 is a plot of cislunar electron content above the plasmapause as a function of time. It was arrived at by subtracting the 5-day running mean value of Syncom-Faraday deduced ionospheric content and calculated protonospheric content from the total value as measured by the radar. In other words, it is the amount left over after subtracting the two lower curves from the upper one of Fig. 5. It is very obvious here that daytime and nighttime behavior are quite different and that, in the anti-solar direction, the content above the plasmasphere is much greater than when looking toward the sun. One interpretation of this content is shown on the right-hand abscissa where columnar content has been divided by radar path length to give an average volume density.

The points in Fig. 6 are plotted in polar coordinates in Fig. 7 to more clearly demonstrate the results. The directional dependence of columnar content above the plasmapause is an outstanding feature of this figure. The average content between 0900 and 1500 hours is $0.04 \times 10^{16}$ electrons $\mathrm{m}^{-2}$ while between 2100 and 0300 hours; it is $4.9 \times 10^{16}$ electrons $\mathrm{m}^{-2}$.

\section{Discussion}

Examination of the experimental data makes it clear that electron content between the ionosphere and the moon is much larger in the antisolar than in the solar direction. Even if the estimated errors combined 


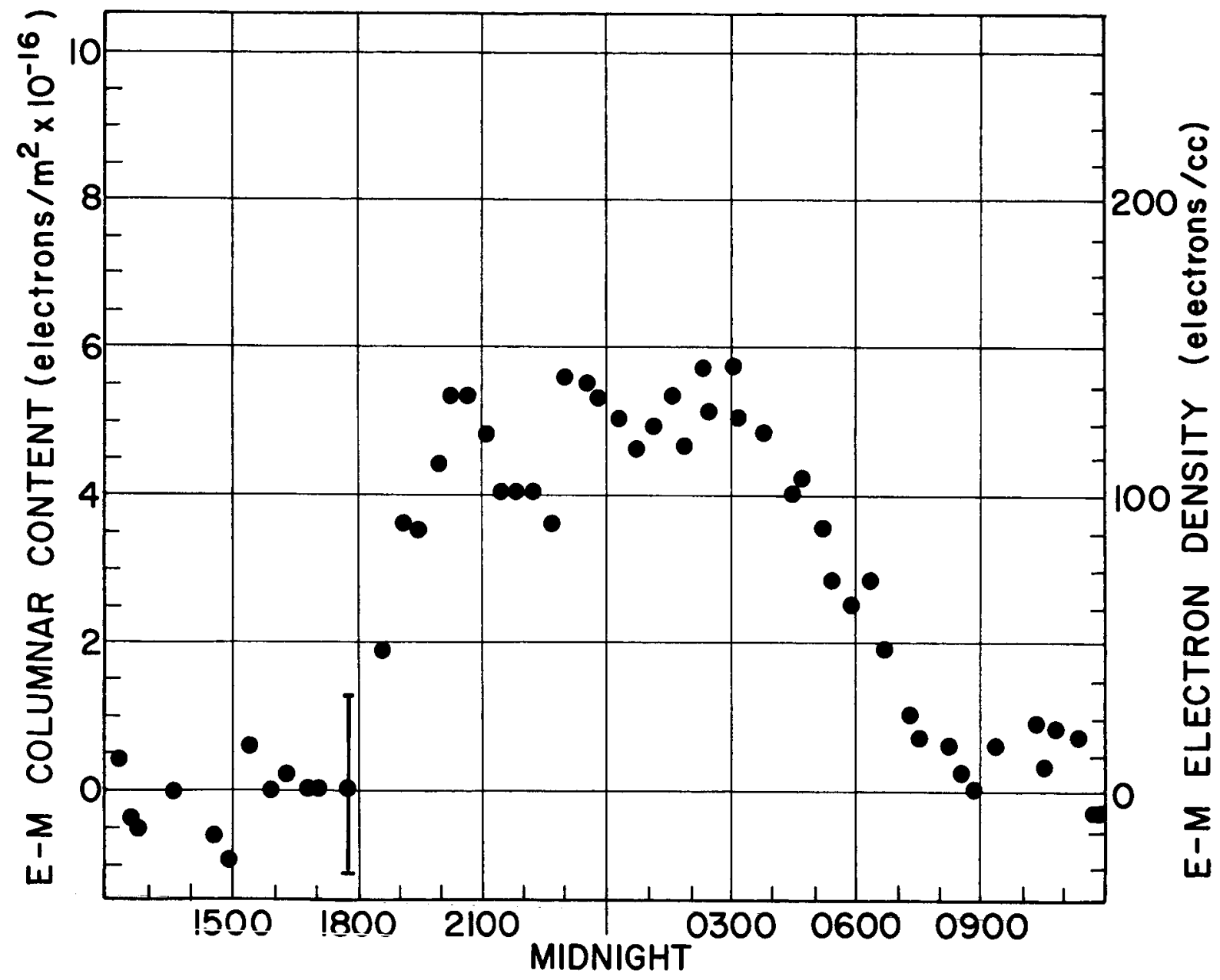

FIG. 6. FIVE DAY RUNNING MEAN OF COLUMNAR CONTENT AND ELECTRON DENSITY BETWEEN THE PLASMAPAUSE AND THE MOON. 


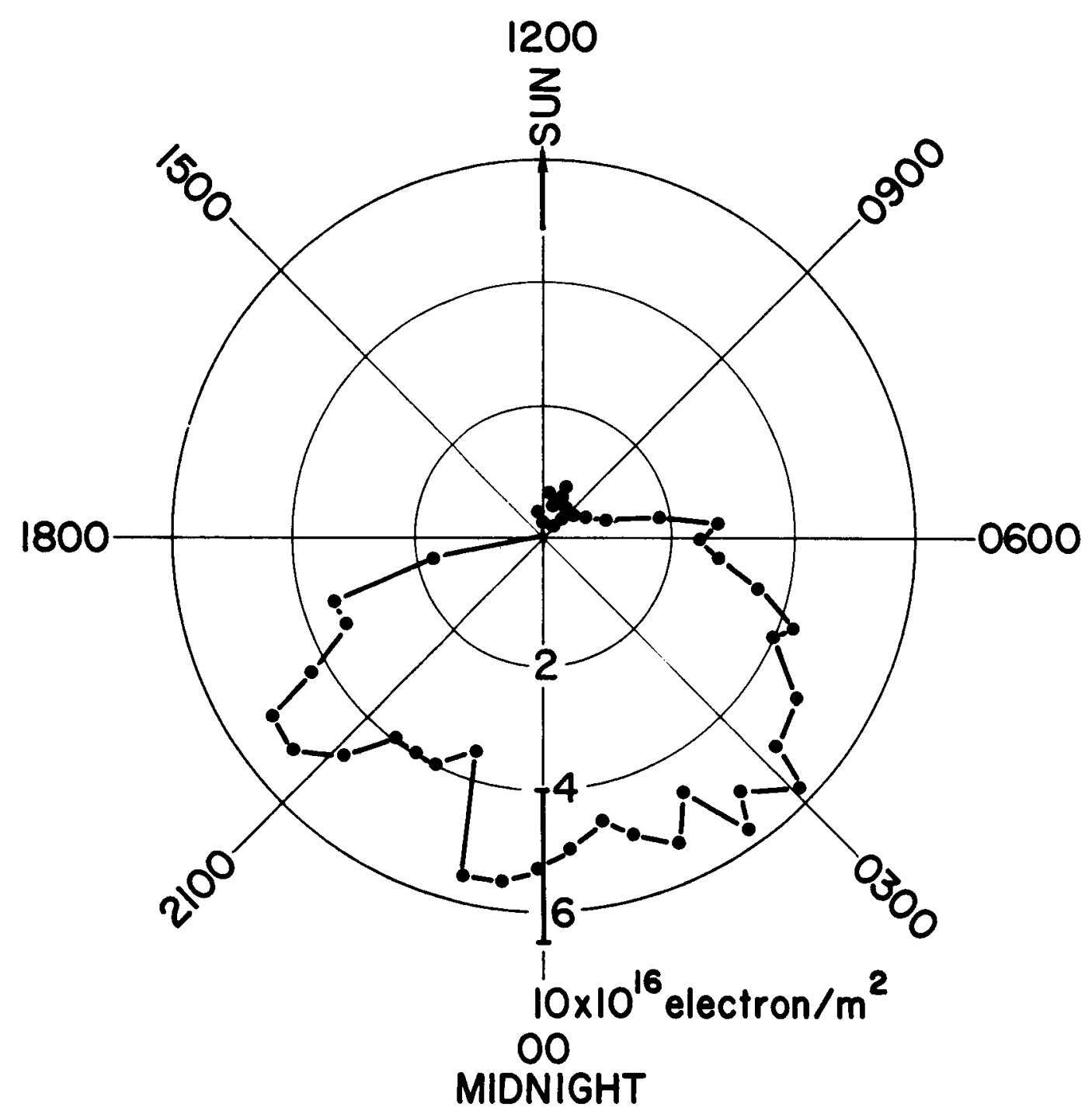

FIG. 7. FIVE DAY RUNNING MEAN OF PLASMAPAUSE-TO-MOON COLUMNAR CONTENT PLOTTED IN GEOCENTRIC SOLAR ECLIPTIC COORDINATES. 
to reduce the content in the anti-solar direction alone, this directional behavior would still be evident.

The radar and syncom results are individually straightforward enough that the error limits assigned are thought to be realistic. The major uncertainty still lies in the protonosphere where no direct measurements through the region have been made. Recent advances in whistler technique and the availability of 1965 data have made it possible to calculate the portion between $4000 \mathrm{~km}$ and the knee with a high degree of accuracy [Angerami, 1966; Carpenter, 1966; Angerami and Carpenter, 1966]. These calculations invariably show that, independent of radar path zenith angle and knee position, content between $4000 \mathrm{~km}$ and the knee is less than $1 \times 10^{16}$ electrons $\mathrm{m}^{-2}$. Thus, the largest contribution to the total protonosphere calculation lies in the 1000 to $4000 \mathrm{~km}$ region where no direct measurements are available for the period in question.

Confidence in the contents calculated for the $1000 \mathrm{~km}$ to $1.5 \mathrm{R}_{\mathrm{E}}$ portion of the path are highest in the anti-solar direction where the radar path closely parallels or crosses the equatorial plane. This confidence is enhanced by the knowledge that the $1000 \mathrm{~km}$ densities and temperatures are known and that the diffusive equilibrium model adequately describes behavior in this region [Angerami and Carpenter, 1966]. In the solar direction, however, the protonospnere computation is more difficult for three reasons: 1) while the $1000 \mathrm{~km}$ density is still known, the radar path is always well above the equatorial plane, 2) it is difficult to assign a realistic temperature to the medium, and 3) the dominance of hydrogen can no longer be assumed. However, the fact that the interplanetary density remaining in the solar direction is a small 
number and that the positive and negative excursions of this number lie within the error limits gives assurance that the numbers derived are meaningful. This same fact adds considerable strength to conclusions reached for the anti-solar direction.

Comparison of the previously mentioned lunar radar work by Yoh et al [1966] with additional protonosphere calculations further reinforces these conclusions. In the above paper, day- and night-side contents were deduced from combined Doppler and Faraday records. The data were taken during the winter of 1964 when the daytime path was nearly parallel to the equatorial plane and the nighttime path was well above it--just the reverse of the situation shown in Fig. 2. Calculation of protonosphere electron contents for this geometry yields 5 and $2 \times 10^{16}$ electrons $\mathrm{m}^{-2}$ for the day and night sides respectively. When these numbers are subtracted from the radar results, the day-side content becomes very small, while that remaining in the anti-solar direction is between 3 and $4 \times 10^{16}$ electrons $\mathrm{m}^{-2}$.

\section{Conclusions}

These radar results in the solar direction agree well with whistler data which suggest a very low density beyond the knee [Carpenter, 1966] and with the plasma [Wolfe, 1966; Lazarus et al, 1966] and radio propagation [Eshleman et al, 1966] experiments on board the Pioneer VI spacecraft. The general agreement between the 3 very different Pioneer measurements is striking with an average interplanetary electron number density of less than $10 \mathrm{~cm}^{-3}$ emerging strongly. The average from radar measurements between the hours of 0900 and 1500, though very rough, is $11 \pm 30 \mathrm{~cm}^{-3}$. 
In the anti-solar direction, the situation is considerably different. Based upon present understanding and with the knowledge that it is imposstble to specify distribution along the path, the conclusion to be drawn from the data is that the average columnar content in the magnetospheric tail from approximately $4 \mathrm{R}_{\mathrm{E}}$ to the moon is $4.9 \pm 1.2 \times 10^{16}$ electrons $\mathrm{m}^{-2}$. If this content were spread evenly along the path, it would imply an electron number density of $120 \pm 30 \mathrm{~cm}^{-3}$. Confidence in the protonosphere calculation is highest in this direction and is bolstered by the fact that the more complicated dayside model yields reasonable results. Careful consideration of the measurement techniques and detailed analysis of the plasmasphere's contribution to the overall content has failed to reveal any more reasonable interpretation of the data. This result does not fit well with recent theoretical work [Dessler and Michel, 1966], for it is difficult to explain such a high concentration, either by transporting plasmasphere electrons into the tail or by compressing solar plasma. Magnetometer measurements on IMP I Identify a neutral sheet region [Ness, 1966] and it has been demonstrated [Axford et al, 1965] that such a region requires the presence of plasma to maintain pressure equilibrium. The particle densities possible using these theories, however, are 3 to 10 times less than the radar measurements described here suggest

Since the difference between theory and measurement is so large, It is important to note that the weakest facet of the radar result lies in the protonosphere calculation between 1000 and $4000 \mathrm{~km}$. While the $1000 \mathrm{~km}$ densities are well known at certain times, there are very few direct measurements through the region and the density behavior with 
height is not well enough understood to completely eliminate the possibility that a fraction of the observed content could be found within the protonosphere. In all likelihood, it will be a small fraction because if the assumed electron number density of $6 \times 10^{3} \mathrm{~cm}^{-3}$ were to extend undiminished to $4000 \mathrm{~km}$, the integrated content would be only $1.8 \times 10^{16}$ electrons $\mathrm{m}^{-2}$ while the model used yields $1.2 \times 10^{16}$ electrons $\mathrm{m}^{-2}$. It is, however, important that direct experimental results such as those obtainable by various radio propagation experiments on board the EGO and ATS earth satellites be obtained. Such measurements, when combined with whistler and Alouette data, could completely and accurately define this region.

On the other hand, the close agreement between theory and measurement on the day side strongly suggests that the anti-solar proponosphere model is correct and that present plasma models of the magnetospheric tail are inadequate to explain its observed characteristics. 


\section{Acknowl edgment}

I wish to thank Professor Von R. Eshleman who laid much of the theoretical foundation for the radar work and guided the research with frequent suggestions and discussions. I am also indebted to Drs. J. J. Angerami, D. L. Carpenter and A. V. daRosa for their contributions to, and comments on the protonosphere calculations. The lunar orbit computations were accomplished using a program provided by Dr. Tor Hagfors of M.I.T. Lincoln Laboratory and the Syncom Faraday data were obtained from F. L. Smith, III. Special credit is due to Mr. W. E. Faulkerson for his ingenuity and thoroughness in constructing and calibrating the frequency generation and exciter equipment and for his perserverence in operating and maintaining the entire radar facility.

Research in this paper was supported by the National Aeronautics and Space Administration and the National Science Foundation. 


\section{References}

Angerami, J. J., Private communication, 1966.

Angerami, J. J. and D. Lo Carpenter, Whistler studies of the plasmapause in the magnetosphere (2), J. Geophys. Res., 11, 711-725 1966.

Axford, W。 I。, H. E. Petschek, G. L. Siscoe, Tail of the magnetosphere, J. Geophys。Res。, 70, 1231-1236, 1965.

Barry, G。 H., and R. B。 Fenwick, HF measurements using extended chirpradar techniques, Tech。Rpt。no.103, Contract Nonr-225(64), SU-SEL-65-058, Stanford Electronics Laboratories, stanford, California, June 1965.

Brace, L。 H。, Private communication, 1966.

Carpenter, D。 L。, Whistler studies of the plasmapause in the magnetosphere (1), J. Geophys。Res., I1 $, 693-709,1966$.

Chan, K. Lo, L. Colin, and J. O. Thomas, Electron densities and scale heights in the topside ionosphere: Alouette I observations over the American continents, NASA SP-3027, National Aeronautics and Space Administration, Washington, D。C., 1966.

Colin, L. Private communication, 1966.

daRosa, A. V., Private communication, 1966.

Dessler, $A_{\circ} J_{0}$, and $F_{\circ} C_{0}$ Michel, Pl asma in the geomagnetic tail, J. Geophys. Res. $71,1421-1426,1966$.

Eshleman, V。 R。, P. B. Gallagher, and R。C. Barthle, Radar methods of measuring the cislunar electron density, J. Geophys. Res., 65, 3079-3086, 1960 .

Eshleman, V。 $\mathbf{R}_{0}$, O。 $K_{\circ}$ Garriott, R。 L. Leadabrand, A. M. Peterson, H. T. Howard, $R_{0} L$. Koehler, $R_{0} A$. Long, and $B$ 。 $B$. Lusignan, The interplanetary electron number density from preliminary analysis of the Pioneer 6 radio propagation experiment, J. Geophys. Res., 71, 3325-3327, 1966 .

Garriott, O。K., F. L. Smith, III, P。C. Yuen, Observations of ionospheric electron content using a geostationary satellite, Planetary and Space Sci., 13, 829-838, 1965.

Hagfors, T., Private communication, 1966.

Howard, H。 T。, V。 R。 Eshleman, G。H。Barry, and R. B. Fenwick, Radar measurements of the cislunar electron content, J. Geophys. Res., 70, 4357-4363, 1965 . 
Lazarus, A. J., H. S. Bridge, and J. Davis, Preliminary results from the Pioneer 6 M.I.T. plasma experiment, J. Geophys. Res., 71, 3787$3790,1966$.

Ness, N. F., The earth's magnetic field: A new look, Science, 151, 1041-1052, 1966.

Scarf, F. L., Effect of coherent density fluctuations on the radar determination of total electron content, J. Geophys. Res., 71, 2709$2713,1966$.

Smith, F. L., III, Private communication, 1966.

Thomas, J. O., M. J. Rycroft, and L. Colin, Electron densities and scale heights in the topside ionosphere: Alouette I observations in midlatitudes, NASA SP-3026, National Aeropautics and space Administration, Washington, D. C., 1966.

Wolfe, J. H., R. W. Silva, D. D. McKibbin, and R. H. Mason, The compositional, anisotropic, and nonradial flow characteristics of the sol ar wind, J. Geophys. Res., 71, 3329-3335, 1966.

Yoh, P. H. T. Howard, B. B. Lusignan, and V. R. Eshleman, Lunar radar measurements of the earth's magnetospheric wake, J. Geophys. Res., 71 , 189-193, 1966. 